\title{
A vehicle detection algorithm based on the multi-sensors fusion and multi-vision-features
}

\author{
Jin $\mathrm{Lu}^{1, \mathrm{a}}$, Kang $\mathrm{Xiao}^{2, \mathrm{~b}}$ and Wu Yue $\mathrm{W}^{3, \mathrm{c}}$ \\ ${ }^{1}$ No.4 Yard, Huaishuling, Fengtai District, Beijing, China \\ ${ }^{2}$ No.4 Yard, Huaishuling, Fengtai District, Beijing, China \\ ${ }^{3}$ No.4 Yard, Huaishuling, Fengtai District, Beijing, China \\ ajinlubit6@126.com, bkangxiaotop1@126.com, 407811141@qq.com
}

Keywords: intelligent vehicle; vehicle detection; multi-vision features; space alignment

\begin{abstract}
With the importance of drive assistant system in intelligent vehicle, the vehicle detection fusing multi-sensors and multi-vision features is provided. The vehicle detection algorithm is composed of two steps. Firstly, the vision sensor and millimeter wave radar sensor are used, and with space transform between image coordinate and radar coordinate, the space alignment between two sensors is realized. Then, the vehicle is detected by fusing the different vision features including bottom shadow, symmetry, left and right edges. The performance of the detection algorithm is verified under simple and complex environment, and the results show the vehicle detection algorithm is valid and feasible.
\end{abstract}

\section{Introduction}

With the rapid glowing of vehicle, the traffic accidents happen frequently, and cause serious casualties and financial losses. So that developing the effective drive assistance system has given rise to a lot of attention lately. The reliable vehicle detection is a very significant part of drive assistant system[1].

The environment information can be obtained by the sensors installed onto the intelligent vehicle. The vision sensor can gain vehicle contour information in sensing region, meanwhile, the millimeter wave radar can detect moving vehicle, and provide the radial range and accurate velocity information[2]. So that fusing the two complementary sensors measurement will be able to raise the information wholeness of vehicle detection, in other words, the fusion method can provide the comprehensive vehicle detection information including either contour size or radial range and related velocity[3].

The vehicle detection method based on these two sensors generally is divided into the two parts: space alignment about two sensors, and the verification about aligned target in the image plane. The traditional space alignment need to estimate the interior and exterior parameters between different sensors. Sugimoto et al.[2] considered the potion which has maximum reflection intensity as the related target position in the image plane. However, the maximum reflection intensity need a special tool to obtained and is not be explained carefully. Zieke et al[4].used the intensity symmetry to determine the target vehicle position. Kim et al[5]. made use of the vehicle bottom shadow for generating the region of interesting, and determining the vehicle existence through finding the vehicle symmetry. Nevertheless, the single feature to verify target vehicle position is very difficult for reliable vehicle detection.

The positive results included are listed as follows: firstly, space alignment projecting the target from millimeter wave radar onto the image captured from vision sensor is realized in order to reduce the sequent load, and don't use a special tool in this process. Secondly, a segmentation method based on the modified otsu algorithm is proposed in order to segment vehicle bottom shadow. Finally, For overcoming the single feature limitation, the vehicle detection algorithm fusing multi-vision features including vehicle bottom shadow, vehicle symmetry, and the left and right edge of vehicle. The 
effective performance obtained by fusing the space alignment and vehicle detection is obtained in the different scenarios.

\section{Multi-sensors Space Alignment}

The space alignment can be performed according to the corresponding reference target point sets between image coordinate of vision sensor and the millimeter wave radar coordinate. The point sets are composed of the reference target point received through millimeter wave radar scanning and the centroid of reference target in image coordinate. The relationship between these corresponding point sets can be expressed through the Eq. 1.

$$
\left[\begin{array}{c}
u_{c} \\
v_{c} \\
1
\end{array}\right]=N\left[\begin{array}{c}
r_{m} \sin \theta_{m} \\
r_{m} \cos \theta_{m} \\
1
\end{array}\right]=\left[\begin{array}{lll}
N_{11} & N_{12} & N_{13} \\
N_{21} & N_{22} & N_{23} \\
N_{31} & N_{32} & N_{33}
\end{array}\right]\left[\begin{array}{c}
r_{m} \sin \theta_{m} \\
r_{m} \cos \theta_{m} \\
1
\end{array}\right] .
$$

Where the $r_{m}$ denotes the range of reference target in millimeter wave radar coordinate; the azimuth of the reference target is shown by $\theta_{m}$; the $N$ is space transformation from millimeter wave radar coordinate to image coordinate.

The $\left(u_{c}, v_{c}\right)$ is the centriod of the reference target in image coordinate. So that The $N$ can be estimate by the least square method including not less than five corresponding point sets.

With the $f\left(u_{i}, v_{i}\right)$ representing the pixel grey value in image plane, the centriod $\left(u_{c}, v_{c}\right)$ of the reference target can be shown by the Eq. 2 .

$$
u_{c}=\frac{\sum_{u_{i}} \sum_{v_{i}} u_{i} f\left(u_{i}, v_{i}\right)}{\sum_{u_{i}} \sum_{v_{i}} f\left(u_{i}, v_{i}\right)}, v_{c}=\frac{\sum_{u_{i}} \sum_{v_{i}} v f\left(u_{i}, v_{i}\right)}{\sum_{u_{i}} \sum_{v_{i}} f\left(u_{i}, v_{i}\right)} .
$$

Let $U_{c}=\left[\begin{array}{lll}u_{c}^{1} & \mathrm{~L} & u_{c}^{n}\end{array}\right]^{T}, V_{c}=\left[\begin{array}{lll}v_{c}^{1} & \mathrm{~L} & v_{c}^{n}\end{array}\right]^{T}, N_{j}=\left[\begin{array}{lll}N_{j 1} & N_{j 2} & N_{j 3}\end{array}\right]^{\mathrm{T}}, I_{n \times 1}=\left[\begin{array}{lll}1 & \mathrm{~L} & 1\end{array}\right]^{T}$, Therefore, the space transformation computed through the least square method can be shown by the Eq. 3, where the $N_{1}, N_{2}, N_{3}$ is listed by the Eq. 4 .

$$
\begin{aligned}
& N=\left[\begin{array}{lll}
N_{1}^{T} & N_{2}^{T} & N_{3}^{T}
\end{array}\right]^{T} . \\
& N_{1}=\left(P P^{\mathrm{T}}\right)^{-1} P^{\mathrm{T}} U_{c}, N_{2}=\left(P P^{\mathrm{T}}\right)^{-1} P^{\mathrm{T}} V_{c}, N_{3}=\left(P P^{\mathrm{T}}\right)^{-1} P^{\mathrm{T}} I_{n \times 1} .
\end{aligned}
$$

According to the range and azimuth information on the target observed by millimeter wave radar, the space transformation $N$ is used to project the target information onto the image plane, and realize the space alignment of millimeter wave radar sensor and vision sensor.

\section{Modified Dual-thresholds otsu Method}

The traditional otsu method is a global threshold selection method, and obtains the optimal threshold through maximizing the variance between object region and background region[6]. However, this method only has the good segmentation to the very simple environment. The dual-thresholds otsu method is an extension of the otsu method, but it mainly has a disadvantage, that is the course to resolve optimal dual-thresholds needs much time because it has to traverse all possible dual-thresholds. Meanwhile, the generic algorithm $(g a)$ simulate the genetic evolution process of the natural biological, and essentially is a global optimization search algorithm[7]. Therefore, the dual-thresholds can be solved by combining the $g a$, then improve the dual-thresholds otsu method efficiency. So a modified dual thresholds otsu method is presented, and is described as follows:

(1) Initialize the segmented image regions numbers $n_{r}\left(n_{r}=3\right)$, the $g a$ parameter including population size $p_{s}$, maximal iteration count $I_{m}$, crossover probability $p_{c}$, mutation probability $p_{m}$.

(2) Consider the dual-thresholds segmenting image as the chromosome of $g a$, and encode with eight bit binary code, then initialize population $p(t)$ composed of the chromosomes, and let $t=1$. 
(3) Decode the population in order to express the dual-thresholds with two real numbers. Then compute the population fitness fit with the region variance of dual-thresholds OTSU method, and is shown by Eq. 5.

$$
\text { fit }=\sigma_{B}^{2}=w_{1}\left(\mu_{1}-\mu_{T}\right)^{2}+w_{2}\left(\mu_{2}-\mu_{T}\right)^{2}+w_{3}\left(\mu_{3}-\mu_{T}\right)^{2} .
$$

Where $w_{1}, w_{2}, w_{3}$ is the probability of each region; $u_{1}, u_{2}, u_{3}$ is the mean gray of each region; $t h_{1}, u_{T}$ is average gray of image. They can be expressed by the Eq. 6 and Eq. 7. $p_{i}$ is the probability of gray level $i$.

$$
w_{a(a=1,2,3)}=\sum_{i} p_{i}, u_{a(a=1,2,3)}=\sum_{i}\left(i \cdot p_{i}\right) / w_{a} .
$$

Where the range of $i$ in region 1 is from 0 to $t h_{1}$; the range of $i$ in region 2 is from $t h_{1}+1$ to $t h_{2}$; the range of $i$ in region 3 is from $t h_{2}+1$ to $255 . t h_{1}, t h_{2}$ is the threshold corresponding segmented region $w_{1}, w_{2}$, and $w_{3}$.

$$
\mu_{T}=\sum_{i=0}^{255} i p_{i} \text {. }
$$

(4) Generate the new population $p(t)$ through using the selection, crossover maturation operator, and keep back the maximal fitness chromosome of current population to $p(t)$.

(5) Let $t=t+1$, and judge the termination condition: $t \leqslant I_{m}$, if the condition holds, switch to the step (3), else determine the optimal dual-thresholds $b_{t h}$ in the population. Then, segment the image using the $b_{t h}$.

\section{Multi-vision Features Vehicle Detection Method}

Because of the limitation of single vision feature, the multi-vision features should be fused to exactly detect and verify the vehicle size. Three significant vision features are included: the first notable feature is vehicle bottom shadow[8]; the second is vehicle symmetry[9]; and the last is the left and right edges of vehicle[10].

\section{Vehicle Bottom Shadow Detection}

By observing the image intensity, it is well known that the vehicle bottom shadow is darker than other areas on paved road. So the vehicle bottom shadow should be used as the vital feature for detecting the vehicle. So the vehicle bottom shadow detection steps is listed as follows:

(1) Segment image with the modified dual-thresholds OTSU method, and with the space alignment result, find separately the start and end points of the shadow through the Eq. 8 and Eq. 9. $I_{s}$ is vehicle bottom shadow segmentation image.

$$
\begin{aligned}
& I_{s}(v, u-1)-I_{s}(v, u)=255 \& I_{s}(v, u)-I_{s}(v, u+1)=0 . \\
& I_{s}(v, u-1)-I_{s}(v, u)=0 \& I_{s}(v, u+1)-I_{s}(v, u)=255 .
\end{aligned}
$$

(2) Eliminate these start and end points whose pixel value equal to 255 in the column space alignment locates, According to the vehicle bottom shadow continuity.

(3) Detect the vehicle bottom shadow using the maximal width of corresponding start and end points.

The vehicle bottom shadow detection example is shown as Fig. 1.
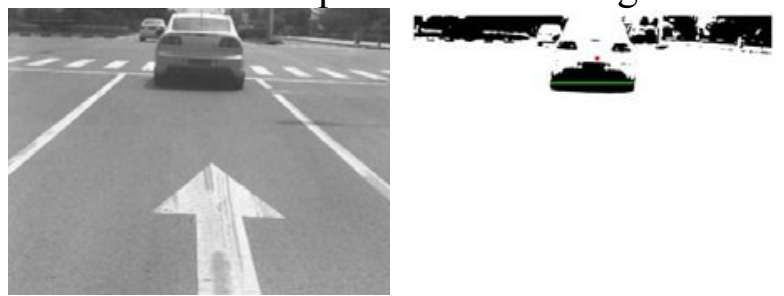

Fig. 1. vehicle bottom shadow detection result.

The left is Original image. The right is image segmentation result; the red cycle is space alignment result; and the green line is the vehicle bottom detection result. 


\section{Vehicle Symmetry Detection}

The vehicle viewed from back is symmetric, so that the vehicle symmetry is also important feature to detect vehicle. The S-channel of HSI color space is not sensitive to the intensity caused by illumination, so that the vehicle symmetry is calculated on the S-channel image. The specific steps are described as follows:

(1) Obtain the S-channel image through color space transformation.

(2) Set vehicle bottom shadow width area as the scope of calculating vehicle symmetry to reduce processing load.

(3) Determine vehicle symmetry with the Eq. 10.

$$
\operatorname{sym}=\arg \min \left(\sum_{u=u_{s l}}^{u_{s v}} \sum_{v=1}^{v_{s}} \sum_{\Delta=1}^{w_{s} / 2}|S(v, u+\Delta)-S(v, u-\Delta)|\right) \text {. }
$$

where $S$ is the S-channel value; ws is vehicle bottom shadow width; $v_{s}$ is the row value of vehicle bottom shadow; $u_{s l}$ and $u_{s r}$ are the left column value and right column value of vehicle bottom shadow.

The Fig. 2 shows the vehicle symmetry detection results.

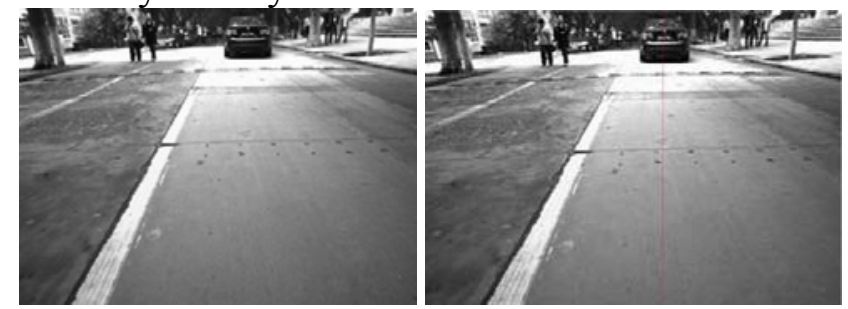

Fig. 2. vehicle symmetry detection result.

The left is Original image. The right is vehicle symmetry marked with the red line.

\section{Vehicle Left and Right Edges detection}

It is well known that the vehicle has a pair of edge features: the left and right edges.

So they also should be used as a due to detect vehicle. To reduce the noise affection, the above vehicle bottom shadow width and vehicle symmetry should be used in order to detect them accurately. The steps to detect them include mainly:

(1) Calculate the horizon difference values in each column of image, then get the horizon difference projection.

(2) In vehicle bottom shadow width area, compute the maximal value on both sides of the obtained vehicle symmetry.

(3) Compare the obtained two values, Determine the column where has a larger values as the left of right edge, and decide the other vehicle edge according to the symmetry.

The vehicle left and right edges detection result is shown as Fig. 3.
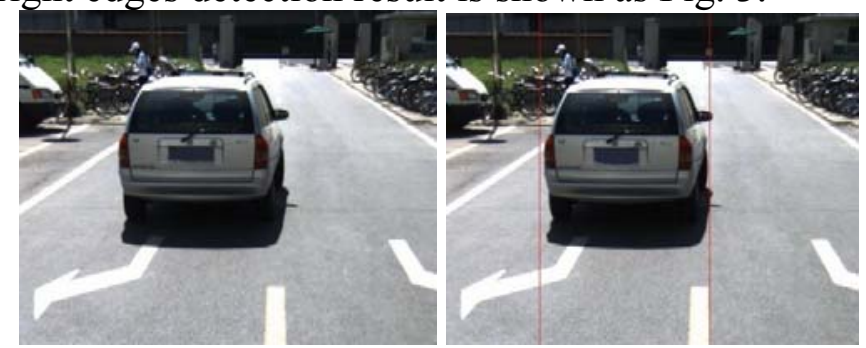

Fig. 3. vehicle left and right edges detection result.

The left is Original image. The right is the left and right edges labeled with red line.

\section{Experiment Results and Analysis}

The vehicle bottom shadow segmentation has a very important role for detecting vehicle. To explain the efficiency of the modified dual-thresholds OTSU method, the misclassification error $\left(e_{m}\right)[11]$ is introduced and shown by the Eq. 11 . 


$$
e_{m}=1-\frac{\left|B_{o} \cap B_{T}\right|+\left|F_{o} \cap F_{T}\right|}{\left|B_{o}\right|+\left|F_{o}\right|} \text {. }
$$

Where $B_{o}$ and $F_{o}$ is separately the background and frontground area pixels set of segmentation image obtained by manual threshold; $B_{T}$ and $F_{T}$ is separately the background and frontground area pixels set of segmentation image obtained by different algorithms.

The essence of $e_{m}$ is the probability of the mistaken pixels, and its value ranges from 0 to 1 . To these images including simple background and complex background, the statistic result of $e_{m}$ got by the different algorithms is shown as Fig. 4. The blue hexagon line represents the result of otsu; the line with green cycle is the result of modified dual-thresholds otsu method. With the statistic result, the average $e_{m}$ is calculated, and is respectively 0.6737 and 0.1433 . Therefore, the conclusion is that the modified dual-thresholds otsu method has better applicability.

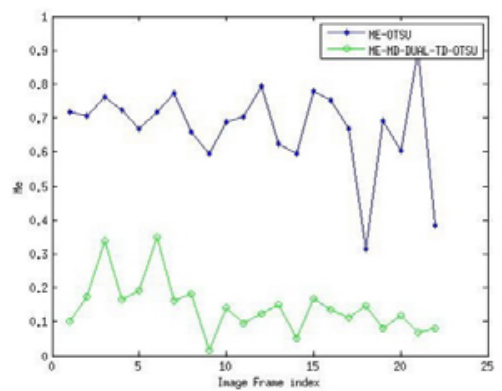

Fig. 4. Statistic result of $e_{m}$

The vehicle detection algorithm also has been used in different environment with good detection results. The environment includes the simple background and complex background. With the analysis about the test results, the accuracy rate for detecting successfully the vehicle reach up about $91 \%$, and the detection time reduces about $30 \%$. That is to say that the vehicle detection algorithm improves the detection accuracy through fusing multi-sensors and multi-vision features, and also reduce the calculated load. The detection results in different environment are shown as Fig. 5 and Fig. 6.
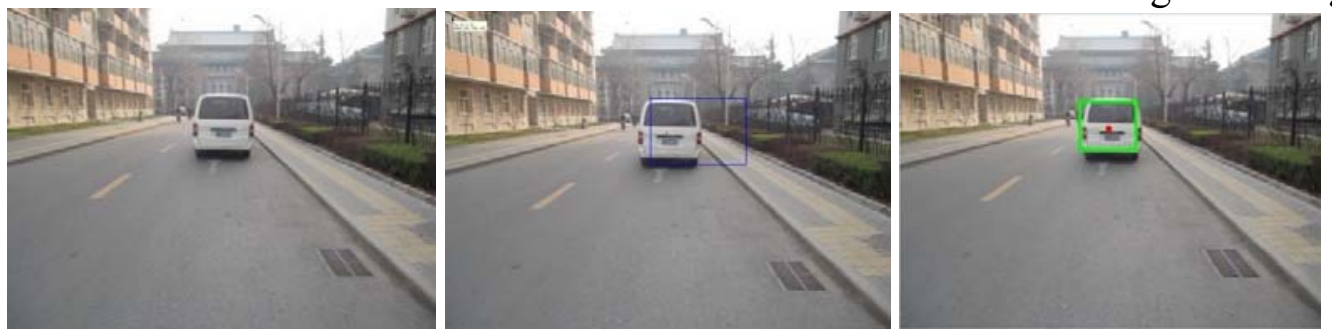

Fig. 5. Vehicle detection results in simple background environment
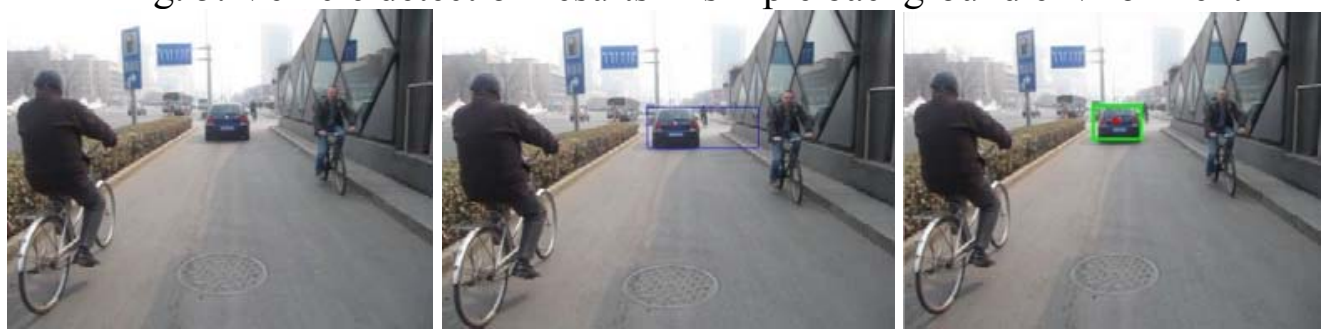

Fig. 6. Vehicle detection results in complex background environment

In Fig. 5 and Fig. 6, the left is original image, the middle and the right are the detection results of different algorithms. The red circular point is the space alignment result about data information captured by millimeter wave radar sensor and vision sensor; the blue rectangle is detection results of the algorithm based on otsu and single symmetry vision feature; the green rectangle is detection result of the present vehicle detection algorithm. So it is can be seen that the proposed vehicle detection algorithm is very effective to determine the vehicle. 


\section{Summary}

A vehicle detection algorithm based on multi-sensor and multi-vision features is proposed. The space alignment obtains the space transformation, and realizes space conversion from millimeter wave radar coordinate to image plane. The multi-vision features of vehicle: the vehicle bottom shadow, vehicle symmetry, and the left and right edges of vehicle are used to determine vehicle. For the vehicle bottom shadow, the modified dual-thresholds otsu method is provided, and solves quickly the optimal dual-threshold through the generic algorithm with global search, and meanwhile, combining the segmented result and space alignment result, the vehicle bottom shadow is abstracted with the shadow continuity. Basis on the width of vehicle bottom shadow, the vehicle symmetry is calculated in S-channel of HSI color space. Depending on the vehicle bottom shadow width and vehicle symmetry, the left and right edges of vehicle are extracted by counting the horizon difference projection. The experiment results show that the vehicle detection algorithm obtains high accuracy results in the environment including simple background and complex background.

\section{References}

[1] S. M. Li, H. Shen, J. G. Mao: Survey of intelligent vehicles development and its key supporting technologies. Transducer and Microsystems Technologies. Vol. 28(2009), p.1-4.

[2] S. Sugimoto, H. Tateda and H. Takahashi: Obstacle Detection Using Millimeter-wave Radar and Its Visualization on Image Sequence. Proceedings of the 17th International Conference on Pattern Recognition. Vol. 3(2004),p.342-345.

[3] J. Laneurit, C. Blanc and R. Chapuis: Multisensorial data fusion for global vehicle and obstacles absolute positioning. Proc. IEEE Intell. Vehicles Symp. (2003), p.138-143.

[4] T. Zieke, M. Brauckmann and W. V Seelen: Intensity and edge-based symmetry detection with an application to car-following. Imaging Understanding. Vol. 58(1993),p.177-190.

[5] S.Y. Kim, S.Y. Oh and J.K. Kang: Front and Rear Vehicle Detection and Tracking in the Day and Night Times Using Vision and Sonar Sensor Fusion. International Conference on Intelligent Robots and Systems. (2005),p.2173-2178.

[6] N.Otsu: A threshold selection method from gray-level histogram. IEEE Trans. System, Man, and Cybernetics. Vol. 9(1979), p.62-66.

[7] J.H Holland: Adaptation in natural and artificial systems: An introductory analysis with application to biology, control, and artificial intelligence( The University of Michigan Press, Michigan 1975).

[8] B. Dai, Y. Fu, and T. Wu: A Vehicle Detection Method via Symmetry in Multi-Scale Windows. IEEE International Conference on Industrial Electronics and Applications. (2007), p.1827-1831.

[9] S.Xu, Y. Zhao and C. Yu: Vehicle Detection Algorithm Based on Shadow Feature. IEEE International Colloquium on Computing, Communication, Control and Management. (2008),p. 105-109.

[10] L. Tsai, Hsieh, and K. Fan: Vehicle detection using normalized color and edge map. IEEE Transactions on Image Processing. Vol. 16(2007), p.850-864.

[11] H.F. Ng. Automatic thresholding for defect detection. Pattern Recognition Letters. (2006), p.16 44-1649. 